\title{
Editorial
}

\section{La importancia del pensamiento crítico en la administración contemporánea}

\section{SEBASTIÁN C. SANTISTEBAN*}

Pensar críticamente los fenómenos sociales y económicos contemporáneos constituye un soporte fundamental para que la reflexión académica pueda asumir una posición ética y epistemológica valiosa y acorde a los grandes retos de hoy. Esa postura debe servir, además, para explorar los distintos aspectos institucionales y organizacionales de la vida de las organizaciones y apuntar a entender los diferentes modos en que el sujeto se relaciona en su interior a partir de una serie de prácticas hegemónicas y de resistencia. Esto implica, a su vez, definir y problematizar operativamente una teoría acorde del discurso que sirva para superar ciertas interpretaciones internalizadas e institucionalizadas que usualmente desechan todo tipo de pensamiento que no sea de corte positivista y funcionalista a fin a los supuestos básicos del capitalismo moderno.

En este sentido se debe reconocer que tradicionalmente los estudios de la administración y las organizaciones han sido abordados como meros servidores y reproductores directos del sistema hegemónico del capital (Cassis and Minoglou, 2005; Hébert and Link, 2009; Murphy, 1986; Nesterov, 2015). Una posición ética y epistemológica -de naturaleza crítica en el estudio de las organizaciones-resulta muy útil para problematizar, por ejemplo, el supuesto protagonismo de distintas corrientes teóricas y nociones como la psicología de la felicidad en el trabajo, el coaching y las técnicas de autocrecimiento que propenden por una producción más eficiente y ágil (Vidaillet, 2013).

El pensamiento crítico de la administración contemporánea demanda que, en el nivel más básico, se debe reconocer que hay algo fundamentalmente errado en este discurso tanto en la manera que configura a una serie de prácticas incontestables (sociales, políticas, culturales y económicas) como en el campo del conocimiento que lo configura y que consecuentemente debe ser transformado.
COMO CITAR ESTE ARTÍCULO

How to cite this article:

Santisteban, S. (2020). Editorial. La importancia del pensamiento crítico en la administración contemporánea. Revista Perspectiva Empresarial, 7(1), 3-6.

* PhD en Estudios Sociales. Fundación Universitaria CEIPA, Sabaneta, Colombia. E-mail: sebastian.neira@ceipa.edu.co. ORCID: 0000-0002-9789-5984. Google Scholar: https://scholar.google.com/citations?user=2fLQt_wAAAAJ\&l=es. 
En principio, un análisis crítico en este campo del saber debe cuestionar lo que se consideran hechos 'obvios' en los que basa sus acciones y se justifica. Debe revelar, además, los fundamentos políticos e ideológicos que sustentan y forman parte de esa cotidianidad de la administración y la manera en que esta se produce y reproduce estructuralmente. Por último, debe revelar más claramente aquello que es aceptable e inaceptable al interior de las lógicas del discurso institucionalizado y proponer a la vez alternativas (Alvesson and Willmott, 2003; Arnaud and Vidaillet, 2018).

\section{Una teoría crítica de la administración desde el psicoanálisis lacaniano}

Si bien el enfoque lacaniano es ampliamente utilizado por los autores para advertir en contra de cualquier forma de actividad seudorrevolucionaria, rara vez se utiliza para entender la formación de una resistencia respecto a los discursos de poder tal como los promovidos por la teoría tradicional de la administración y las organizaciones. En este sentido el giro eventual del posestructuralismo, que articuló lo que hoy se denomina como estudios críticos del management donde se aborda un amplio espectro de temáticas (Cederström and Hoedemaekers, 2010; Fournier and Grey, 2000; Grey and Willmott, 2005; Jones and Spicer, 2009; Willmott, 1992), no se dio sino hasta principios de los años 90 dada la necesidad de profundizar en teorías de la subjetividad que pudieran dar cuenta del creciente individualismo en la sociedad y el menoscabo de la lucha y consciencia de clase provenientes de la teoría marxista (O'Doherty and Willmott, 2001; Vidaillet, 2013).

Así las cosas, debería señalarse que una posible praxis crítica de la teoría lacaniana reta el conflicto tradicional entre los intereses del Otro social con los del sujeto (individuo). Por ello un análisis lacaniano apunta a revelar fundamentalmente la ficción ética que hace creer en la existencia de dicho conflicto, pues se evidencia que todo deseo surge a partir del Otro y por lo tanto solo puede ser externo e impuesto a pesar de que el sujeto piense lo contrario (Johnsen and Gudmand-Høyer, 2010).

Consecuentemente la dicotomía entre el individuo y el Otro social se desvanece (en su sentido moderno y burgués) que coincide con posturas como las de Jones y Spicer cuando argumentan que la visión radical que tiene Lacan para los estudios organizacionales es la superación del denominado antihumanismo práctico, el cual parte del reconocimiento de que la subjetividad no es solo frágil y cuestionable sino que siempre debe estar cuestionándose a sí misma. No hay respuestas absolutas ni verdades reveladas, pues estructuralmente esto es un producto imposible dadas las fallas mismas del discurso y del lenguaje.

En este sentido resulta importante evidenciar el significativo aumento de estudios críticos en el campo de las organizaciones y la administración abordados desde la perspectiva lacaniana. Trabajos como los de Johnsen y Gudmand-Høyer (2010), quienes investigaron la aplicación del psicoanálisis para la crítica del concepto de 'humanidad' en la administración de recursos humanos; al igual que otras investigaciones que sirven para reconocer las múltiples ventajas y beneficios que este marco teórico puede brindar a un estudio de las organizaciones más elaborado y comprensivo (Fotaki and Harding, 2013; Sköld, 2010; Stavrakakis, 2010) al partir desde las perspectivas del sujeto, el deseo y el goce.

El enfoque crítico lacaniano permite no solo limitarse a denunciar los distintos asuntos problemáticos respecto al inconsciente y el goce, sino también para construir posibles salidas y alternativas (Hoedemaekers, 2010) a los circuitos de producción y poder de los aparatos simbólicos. Pues los ejercicios de narrar la realidad internalizada del trabajo y las organizaciones, por ejemplo, brindan la oportunidad de expresar deseos cuya identificación puede ser usada para que los sujetos rompan con las distintas proyecciones imaginarias que los alienan y a la vez oprimen. Ello hace posible examinar y experimentar las distintas maneras en que dicha falta puede ser utilizada como una forma de confrontación creativa con el Otro social, alienante y opresivo (Driver, 2014; Vanheule, Lievrouw and Verhaeghe, 2003). Este tipo de posturas analíticas debe favorecer el fracaso de esas proyecciones imaginarias (y del sujeto en 
lo fundamental) y debe conectar a la vez con la falta que se produce con la entrada en el lenguaje para, en última instancia, poder desarrollar una capacidad (más) libre y liberadora que en la forma de resignificación subjetiva (Driver, 2009; Hoedemaekers, 2010). Por ello la creación de espacios discursivos alternativos en la teoría de la administración y las organizaciones por medio de los cuales dicha falta puede ser articulada, e incluso amplificada, permitirá a los sujetos experimentarse a sí mismos como sujetos capaces de articular y comprometerse con su propio deseo (Arnaud and Vidaillet, 2018; Harding, 2007).

Finalmente la división estructural del sujeto y las correspondientes consecuencias que lo llevan a apoyarse en distintas estructuras simbólicas que lo oprimen también deben problematizarse. De ahí que un análisis crítico de la teoría de la administración y las organizaciones, además de exponer la coerción impuesta desde el exterior (que también es importante y opera), debe buscar identificar y discutir la violencia impuesta desde adentro puesto que es desde allí que el sujeto trabajador principalmente se ve controlado de acuerdo con un conjunto de valores y principios que el marco institucional le impone y le hace creer que provienen de su propia iniciativa como, por ejemplo, los diferentes niveles requeridos de habilidades y conocimientos, los regímenes disciplinarios, las aplicaciones del tiempo, la búsqueda de la felicidad entre otros. El hecho de que hoy, en las prácticas laborales y de gestión, los trabajadores a menudo sean invitados a "ser simplemente ellos mismos" tan solo subraya la importancia de este problema.

\section{Referencias}

Alvesson, M. and Willmott, H. (2003). Studying management critically. New York, USA: SAGE publications.

Arnaud, G. and Vidaillet, B. (2018). Clinical and critical: The Lacanian contribution to management and organization studies. Organization, 25(1), 69-97.

Cassis, Y. and Minoglou, I.P. (2005). Entrepreneurship in Theory and History: State of the Art and New Perspectives. En Entrepreneurship in Theory and
History (pp. 3-21). London, England: Palgrave Macmillan.

Cederström, C. and Hoedemaekers, C. (Ed.) (2010). Lacan and organization. London, United Kingdom: MPG Books Group.

Driver, M. (2009). Struggling with lack: A Lacanian perspective on organizational identity. Organization Studies, 30(1), 55-72.

Driver, M. (2014). The stressed subject: Lack, empowerment and liberation. Organization, 21(1), 90-105.

Fotaki, M. and Harding, N. (2013). Lacan and sexual difference in organization and management theory: Towards a hysterical academy? Organization, 20(2), 153-172.

Fournier, V. and Grey, C. (2000). At the Critical Moment: Conditions and Prospects for Critical Management Studies. Human Relations, 53(1), 7-32.

Grey, C. and Willmott, H. (2005). Critical management studies: A reader. Oxford, United Kingdom: Oxford University Press.

Harding, N. (2007). On Lacan and the 'becoming-ness' of organizations/selves. Organization Studies, 28(11), 1761-1773.

Hébert, R.F. and Link, A.N. (2009). A history of entrepreneurship. London, England: Routledge.

Hoedemaekers, C. (2010). 'Not even semblance': Exploring the interruption of identification with Lacan. Organization, 17(3), 379-393.

Johnsen, R. and Gudmand-Høyer, M. (2010). Lacan and the lack of humanity in HRM. Organization, 17(3), 331-344.

Jones, C. and Spicer, A. (2009). Unmasking the entrepreneur. Cheltenham, United Kingdom: Edward Elgar publishing.

Murphy, A.E. (1986). Richard Cantillon. Entrepreneur and economist. Oxford, United Kingdom: Clarendon Press.

Nesterov, D. (2015). History of Microsoft. New York, USA: SUNY State University. 
O'Doherty, D. and Willmott, H. (2001). Debating Labour Process Theory: The Issue of Subjectivity and the Relevance of Poststructuralism. Sociology, 35(2), 457-476.

Sköld, D.E. (2010). The other side of enjoyment: Short-circuiting marketing and creativity in the experience economy. Organization, 17(3), 363-378.

Stavrakakis, Y. (2010). Symbolic authority, fantasmatic enjoyment and the spirits of capitalism: Genealogies of mutual engagement. En Cederström, C. and Hoedemaekers, C. (Ed.), Lacan and organization (pp. 59-100). London, United Kingdom: MPG Books Group.

Vanheule, S., Lievrouw, A. and Verhaeghe, P. (2003). Burnout and intersubjectivity: A psychoanalytical study from a Lacanian perspective. Human Relations, 56(3), 321-338.

Vidaillet, B. (2013). Book Review: Bénédicte Vidaillet reviews Lacan and organization. Organization, 20(2), 324-327.

Willmott, H. (1992). Critical management studies. New York, USA: SAGE publications. 